\title{
Allosteric competitive inactivation of hematopoietic CSF-1 signaling by the viral decoy receptor BARF1
}

\author{
Jonathan Elegheert ${ }^{1}$, Nathalie Bracke ${ }^{1,5}$, Philippe Pouliot ${ }^{2}$, Irina Gutsche ${ }^{3}$, Alexander V Shkumatov ${ }^{4,5}$, \\ Nicolas Tarbouriech $^{3}$, Kenneth Verstraete ${ }^{1}$, Anaïs Bekaert ${ }^{1,5}$, Wim P Burmeister ${ }^{3}$, Dmitri I Svergun ${ }^{4}$, \\ Bart N Lambrecht ${ }^{2}$, Bjorn Vergauwen ${ }^{1} \&$ Savvas N Savvides $^{1}$
}

\begin{abstract}
Hematopoietic human colony-stimulating factor 1 (hCSF-1) is essential for innate and adaptive immunity against viral and microbial infections and cancer. The human pathogen Epstein-Barr virus secretes the lytic-cycle protein BARF1 that neutralizes hCSF-1 to achieve immunomodulation. Here we show that BARF1 binds the dimer interface of hCSF-1 with picomolar affinity, away from the cognate receptor-binding site, to establish a long-lived complex featuring three hCSF-1 at the periphery of the BARF1 toroid. BARF1 locks dimeric hCSF-1 into an inactive conformation, rendering it unable to signal via its cognate receptor on human monocytes. This reveals a new functional role for hCSF-1 cooperativity in signaling. We propose a new viral strategy paradigm featuring an allosteric decoy receptor of the competitive type, which couples efficient sequestration and inactivation of the host growth factor to abrogate cooperative assembly of the cognate signaling complex.
\end{abstract}

The Epstein-Barr virus (EBV) or human herpesvirus-4, a lymphocryptovirus (LCV) species from the $\gamma$-herpesvirus subfamily, is a global human pathogen that establishes lifelong persistent infections in 90-95\% of the human population. Primary lytic replication of EBV in oropharyngeal mucosal epithelium precedes a long-lived latent infection of resting memory $\mathrm{B}$ cells ${ }^{1,2}$. The dual cell tropism of EBV is the basis for the wide spectrum of pathologies associated with EBV infections, which most notably includes the lymphoproliferative disorder infectious mononucleosis ${ }^{2}$.

Like most LCVs, EBV coevolved with its host by developing sophisticated interactions with host molecular targets that mainly exploit molecular mimicry to evade detection and elimination by the human immune system ${ }^{3}$. A striking aspect of the EBV proteomic pool concerns the secretion of the lytic-cycle early protein BARF1 (BamHI-A rightward frame-1), which, like BCRF1 (Bam HI-C rightward frame-1, also known as vIL-10), is a product of a pool of transcripts that occur only in LCV infection in higher primates ${ }^{4}$. Expression and secretion of BARF1 is highly associated with epithelial malignancies, including nasopharyngeal carcinoma and gastric carcinoma ${ }^{5,6}$, and elicits a strong humoral immune response ${ }^{7}$. Furthermore, BARF1 emerged as a potential oncoprotein because of its transforming and mitogenic behavior in murine fibroblasts, human B cells and monkey epithelial cells ${ }^{8-12}$. However, a controversial role of BARF1 in the transformation of naive human B cells has recently been described ${ }^{13,14}$.
The discovery that BARF1 is a binding protein for the hematopoietic cytokine hCSF-1, a central cytokine in innate and adaptive immunity in humans, established a link to the possible immunomodulatory role of BARF1 (ref. 15). BARF1 was also shown to inhibit the secretion of antiviral interferon (IFN)- $\alpha$ (ref. 13), which, interestingly, is produced by mononuclear cells, as a result of hCSF- 1 activity ${ }^{16}$. Together with IFN- $\gamma$, IFN- $\alpha$ establishes a primary response via CD ${ }^{+}$cytotoxic T-cells and natural killer cells to clear EBV-infected cells ${ }^{17}$, in line with $\mathrm{T}$ cell-mediated adaptive immunity against EBV ${ }^{18}$. hCSF-1 initiates intracellular signaling pathways by activating its cognate receptor, hCSF-1R, a cell-surface class III receptor tyrosine kinase (RTK-III), leading to the proliferation, differentiation and activation of cells derived from the mononuclear phagocytic lineage ${ }^{19}$. Bivalent binding of dimeric hCSF-1 to extracellular immunoglobulin-like domains 2 and 3 of hCSF-1R dimerizes the receptor to prime homotypic receptor-receptor interactions that help establish a cooperative high-affinity complex ${ }^{20}$.

We here present the structural and mechanistic basis of the inactivation of hCSF-1 signaling by BARF1 to achieve immunomodulation. Our studies have exposed a new paradigm for virus-human protein interactions and have deepened our understanding of the molecular and structural determinants of the hCSF-1-hCSF-1R extracellular signaling assembly and its potential targeting for therapeutic purposes.

\footnotetext{
${ }^{1}$ Unit for Structural Biology, Laboratory for Protein Biochemistry and Biomolecular Engineering, Department of Biochemistry \& Microbiology, Ghent University, Ghent, Belgium. ${ }^{2}$ Laboratory of Immunoregulation and Mucosal Immunology, Department of Molecular Biomedical Research, VIB and Ghent University, Ghent, Belgium. ${ }^{3}$ Unit for Virus Host-Cell Interactions, Université Joseph Fourier, European Molecular Biology Laboratory and Centre National de la Recherche Scientifique, Grenoble, France. ${ }^{4}$ Biological Small Angle Scattering Group, European Molecular Biology Laboratory, Hamburg Unit, Deutsches Elektronen-Synchrotron, Hamburg, Germany. ${ }^{5}$ Present addresses: Laboratory of Drug Quality and Registration, Department of Pharmaceutical Analysis, Ghent University, Ghent, Belgium (N.B.); Department of Biological Chemistry and Molecular Pharmacology, Harvard Medical School, Boston, Massachusetts, USA (A.V.S.); Oxyrane, Ghent, Belgium (A.B.). Correspondence should be addressed to S.N.S. (savvas.savvides@ugent.be).
} 
Table 1 Crystallographic data collection and refinement statistics

\begin{tabular}{|c|c|c|c|c|c|}
\hline & $\begin{array}{c}\text { BARF 1-hCSF-1: crystal } \\
\text { form } 1\end{array}$ & $\begin{array}{c}\text { BARF } 1-\text { hCSF-1: crystal } \\
\text { form } 2\end{array}$ & hCSF-1 & BARF1-mCSF-1 & mCSF-1 \\
\hline \multicolumn{6}{|l|}{ Data collection } \\
\hline Space group & $R 3$ & $P 3_{1} 21$ & $P 2_{1}$ & $R 3$ & $P 2_{1} 2_{1} 2_{1}$ \\
\hline \multicolumn{6}{|l|}{ Cell dimensions } \\
\hline$a, b, c(\AA)$ & $235.24,235.24,95.71$ & $218.44,218.44,331.17$ & $61.15,104.49,116.24$ & $234.75,234.75,96.03$ & $36.32,68.69,144.62$ \\
\hline$\alpha, \beta, \gamma\left({ }^{\circ}\right)$ & $90,90,120$ & $90,90,120$ & $90,105.29,90$ & $90,90,120$ & $90,90,90$ \\
\hline Resolution ( $\AA$ ) & $40.00-3.40(3.50-3.40)$ & $40.00-4.40(4.51-4.40)$ & $40.00-2.75(3.00-2.75)$ & $40.00-4.50(4.62-4.50)$ & $40.00-2.80(2.87-2.80)$ \\
\hline$R_{\text {meas }}(\%)$ & $18.6(69.5)$ & $12.4(80.6)$ & $16.0(69.3)$ & $15.4(67.5)$ & $8.9(59.3)$ \\
\hline$I / \sigma I$ & $8.8(2.5)$ & $13.5(2.8)$ & $9.7(2.3)$ & $5.7(1.6)$ & $12.9(2.5)$ \\
\hline Completeness (\%) & $96.4(91.7)$ & $99.4(98.2)$ & 99.5 (98.9) & $94.5(3.2)$ & $98.5(98.7)$ \\
\hline Redundancy & $4.0(3.8)$ & $5.6(5.6)$ & $3.8(3.8)$ & $1.7(1.5)$ & $3.3(3.5)$ \\
\hline \multicolumn{6}{|l|}{ Refinement } \\
\hline Resolution $(\AA)$ & $40.00-3.40$ & $40.00-4.40$ & $40.00-2.75$ & $40.00-4.50$ & $40.00-2.80$ \\
\hline No. reflections & 25,859 & 58,094 & 36,555 & 11,078 & 9,312 \\
\hline$R_{\text {work }} / R_{\text {free }}$ & $0.2314 / 0.2735$ & $0.2395 / 0.2763$ & $0.1373 / 0.1903$ & $0.2226 / 0.2622$ & $0.2097 / 0.2578$ \\
\hline \multicolumn{6}{|l|}{ No. atoms } \\
\hline Protein & 10,537 & 31,549 & 11,438 & 10,591 & 2,369 \\
\hline Ion/glycan & $-/ 156$ & $-/ 864$ & - & $-/ 167$ & 4/- \\
\hline Water & - & - & 169 & - & 28 \\
\hline \multicolumn{6}{|l|}{$B$ factors $\left(\AA^{2}\right)$} \\
\hline Protein & 80 & 217 & 59 & 188 & 60 \\
\hline Ion/glycan & -170 & -1245 & - & $-/ 178$ & 89/- \\
\hline Water & - & - & 37 & - & 47 \\
\hline \multicolumn{6}{|l|}{ R.m.s. deviations } \\
\hline Bond lengths ( $\AA$ ) & 0.003 & 0.006 & 0.002 & 0.003 & 0.003 \\
\hline Bond angles $\left({ }^{\circ}\right)$ & 0.64 & 1.26 & 0.49 & 0.71 & 0.55 \\
\hline
\end{tabular}

Values in parentheses refer to the highest-resolution shell. Each of the reported datasets was obtained from one crystal.

\section{RESULTS}

\section{Architecture and assembly of the BARF1-hCSF-1 complex}

Fractionation of purified recombinant glycosylated BARF1 titrated with excess recombinant hCSF-1 (ref. 20) by field-flow fractionation (FFF) followed by multiangle laser scattering (MALS) resulted in a well-resolved molecular species of $\sim 250 \mathrm{kDa}$ (Supplementary Fig. 1a). Crystallization trials on purified BARF1-hCSF-1 complex led to two crystal forms that yielded X-ray diffraction data sets to $3.4 \AA$ and $4.4 \AA$ (Table 1 ).

The crystal structure of the BARF1-hCSF-1 complex, as determined by molecular replacement using the structure of BARF1 (ref. 21) and the structure of hCSF-1 determined herein (Table 1), unveiled an assembly in which the BARF1 hexamer is decorated by three copies of dimeric hCSF-1 at the periphery of its toroidal structure (Fig. 1a). Dimeric hCSF-1 wedges into a saddle-shaped binding platform projected by the two tandem N-terminal immunoglobulin-like domains of BARF1. Despite the medium resolution of our crystallographic analysis, the electron-density maps allowed visualization and modeling of most side chains and of nearly complete glycan trees lining the inner circle of the BARF1 toroid (Supplementary Fig. 1b,c). Structural analysis of the complex by negative-stain EM and smallangle X-ray scattering (SAXS) confirmed the structural homogeneity of our BARF1-hCSF-1 preparations and corroborated the crystal structure (Fig. 1b,c and Supplementary Table 1). To gain insights into the possible plasticity of BARF1 before and after complex formation, we carried out measurements by SAXS of unliganded BARF1. Comparison of the experimental SAXS curves obtained from a broad range of BARF1 concentrations against X-ray scattering curves calculated from the crystal structure of BARF1 (ref. 21) revealed a large overall discrepancy $\left(\chi^{2}=3.9\right)$ and clear differences at low values of the momentum transfer, indicating large deviations in shape (Fig. 1d and Supplementary Table 1). Starting with the crystal structure of BARF1, the SAXS data could be fitted well $\left(\chi^{2}=1.3\right)$ by normal mode analysis (NMA) ${ }^{22}$ based on an iterative structure-refinement protocol employing linear combinations of two normal modes in each step. The refined model displays a squashed ring structure (Fig. 1d) that contrasts the symmetric toroid of BARF1 in crystal structures of both its unliganded form ${ }^{21}$ and complex with hCSF-1 (Fig. 1a). Thus unbound BARF1 may display some flexibility manifested as alternative conformations, a notion further supported by the increase in $D_{\max }$ and features of the distance distribution function (Fig. 1d).

\section{The BARF1-hCSF-1 interaction site}

The structural trivalency of the BARF1-hCSF-1 complex is accompanied by numerous unexpected findings concerning the strategy adopted by BARF1 to sequester hCSF-1. BARF1 employs the $\beta$-turn between strands D and $\mathrm{E}$ and the $\mathrm{BC}$ loop of its $\mathrm{N}$-terminal immunoglobulin-like domain to create the tandem tips of a structural 'tweezer' that grabs dimeric hCSF-1 at the meeting point of the two head-to-head antiparallel $\beta$-sheets at the hCSF- 1 dimer interface, staying clear of the helical-bundle core of hCSF-1 (Fig. 2a,b). Whereas the two-fold axis of the composite BARF1 binding interface coincides with that of the hCSF-1 dimer, each arm of the tweezers contacts the hCSF-1 dimer asymmetrically, burying $\sim 560 \AA^{2}$ of surface area on one hCSF- 1 subunit and $\sim 300 \AA^{2}$ on the other (Fig. 2a).

Unexpectedly, we found that the BARF1-hCSF-1 interaction site is sterically distinct from the canonical binding site employed by hCSF-1 when binding to its cognate receptor hCSF- $1 \mathrm{R}^{20}$. Superposition of the molecular envelope of the hCSF-1-hCSF-1R complex, as determined by $\mathrm{EM}^{20}$ (EMDB access code EMD-1977) with the BARF1-hCSF-1 
Figure 1 Structural studies of the BARF1hCSF-1 assembly. (a) Ribbon representation of the BARF1-hCSF-1 complex. Hexameric BARF1 (blue) binds three hCSF-1 (yellow) dimers. The oligomannose-type glycan structures are shown in stick-and-surface representation. Prime symbols refer to the second hCSF-1 and BARF1 subunits in their respective oligomers. (b) 2D analysis of the BARF1-hCSF-1 complex by negative-stain EM; characteristic ab initio class averages and corresponding X-ray crystal structure projections Fourier-low-path filtered to $25 \AA$. (c) SAXS analysis of the BARF1-hCSF-1 complex and comparisons with the two crystal forms of the complex. (d) SAXS analysis of unbound BARF1. The unbound BARF1 crystal structure (PDB code 2CH8, blue) and NMArefined model (red) are indicated. The fits from the two models are displaced along the logarithmic axis for better visualization. The inset shows the $P(r)$ function for free and complexed BARF1.

complex, using bound hCSF-1 as a reference shows that a supercomplex can be readily constructed (Fig. 2b). Furthermore, BARF1 establishes two limited and tight clusters of interactions with hCSF-1: those arising from the $\mathrm{DE} \beta$-turn comprising residues $82-86$, and those mediated by the BC loop defined by residues 34-39 (Fig. 2c and Supplementary Table 2). Notably, BARF1 does not interact with any key residue on hCSF-1 participating in the canonical binding site with cognate hCSF- $1 \mathrm{R}^{20,23}$.

The DE $\beta$-turn exclusively contributes side chains to the BARF1-hCSF-1 interaction site. The most elaborate interaction is mediated by Asn85, which establishes bifurcated hydrogen bonds with the main chain oxygen and nitrogen of Thr34 in hCSF-1 (Fig. 2c). The Asn85 side chain orientation is defined by a hydrogen bond with the main chain oxygen of Trp35. The BC loop structure establishes a more varied set of interactions with hCSF-1 (Fig. 2c and Supplementary Table 2). In particular, we highlight Val38, which inserts into the hCSF-1 dimer interface to make contacts with Ile33 and the aliphatic parts of Thr64 and Thr29'. The prime symbol refers to amino acid residues in the second subunit of the hCSF1 dimer. Moreover, the main chain nitrogen of Val38 donates a hydrogen bond to the carbonyl oxygen of Asp63 in hCSF-1, whereas its own main chain oxygen is hydrogenbonded to the main chain nitrogen and side chain of Ser30' in hCSF1 (Fig. 2c). Thus, Val38 mediates an interaction bridge between both hCSF-1 subunits. In addition, the main chain nitrogen atoms of the Val38-Leu40-Gly41 stretch participate in an intricate hydrogenbonding network with Ser30' in hCSF-1. Finally, we note that Arg36 and Arg37 in the BARF1 BC loop are structurally heterogeneous and partially disordered, as was previously observed in unbound BARF1 (ref. 21). However, in one of the four complexes in crystal form 2, Arg37 participates in an intricate salt-bridge network with several hCSF-1 residues (Fig. 2c).

\section{BARF1 binds to hCSF-1 with ultrahigh affinity}

Isothermal titration calorimetry (ITC) measurements obtained by titrating hCSF-1 to BARF1 revealed a strongly enthalpically driven

a
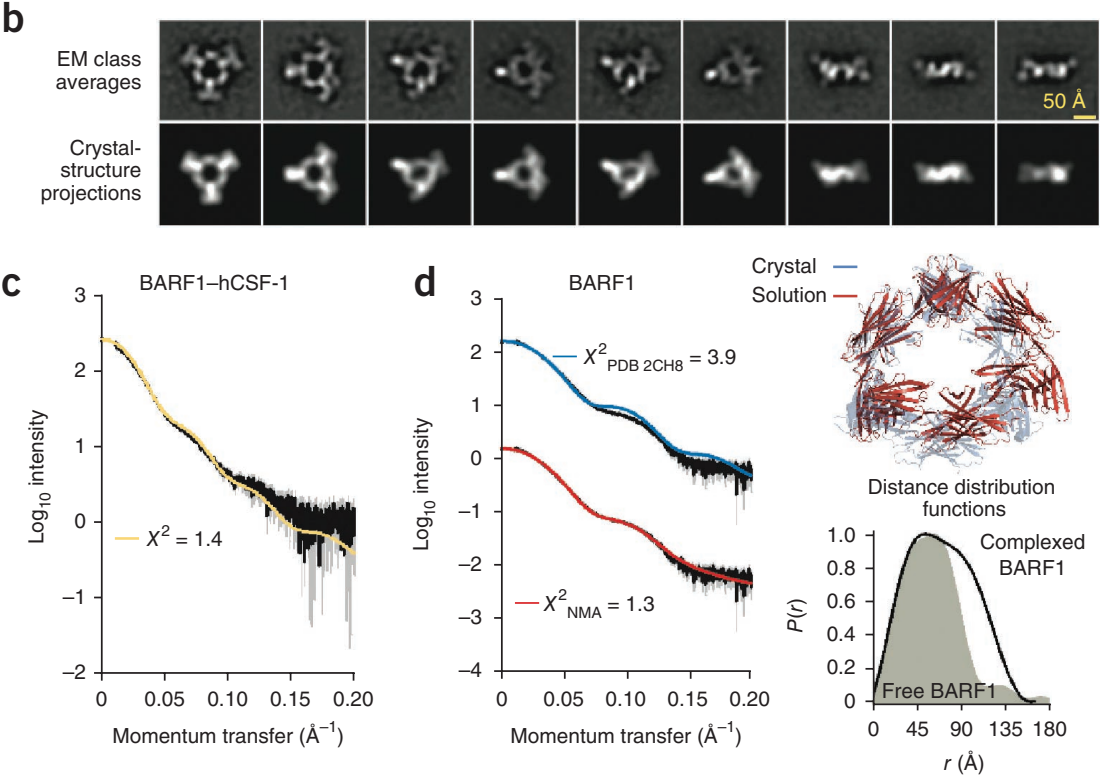

binding event associated with the binding of three hCSF-1 dimers to BARF1 (Fig. 3a), in agreement with our structural observations (Fig. 1). However, the titration transition in the resultant binding isotherm was too steep to allow an accurate calculation of the equilibrium dissociation constant, $K_{\mathrm{D}}$, suggesting an affinity well into the subnanomolar range (Fig. 3a). Using displacement ITC $^{24}$, we titrated BARF1 into an established hCSF-1 $\mathrm{R}_{\mathrm{D} 1-\mathrm{D} 5}-\mathrm{hCSF}-1$ complex, leading to a $K_{\mathrm{D}}=2.50 \mathrm{pM}\left(K_{\mathrm{D}, \mathrm{app}}=1.96 \mathrm{nM}\right)$ (Fig. 3b and Supplementary Table 3), which is at least one order of magnitude tighter than for the interaction of hCSF-1 with cognate hCSF-1R in vivo ${ }^{25}$.

Kinetic characterization of the BARF1-hCSF-1 interaction by surface plasmon resonance (SPR) led to a moderately fast $k_{\text {on }}$ coupled to a very slow $k_{\text {off }}$ dissociation rate constant (Fig. 3c), the latter indicating a mean lifetime of at least $24 \mathrm{~h}$ for the BARF1-hCSF-1 complex. Together, such binding characteristics can be compared with some of the tightest cytokine-receptor interactions measured to date ${ }^{26}$, and they show that BARF1 harbors the traits of a very effective sequestration platform for hCSF-1.

In light of the compact structural footprint of the BARF1-hCSF-1 interaction, we sought to delineate the relative contribution of the $\mathrm{DE}$ $\beta$-turn and BC loop in BARF1. Five amino acids from the BC loop were mutated to alanine, and the thermodynamic binding fingerprint of the mutant was characterized by ITC. This revealed a decrease in the affinity of the complex by a factor of $10^{4}$ (Supplementary Fig. 2a 
Figure 2 BARF 1 targets hCSF-1 through a new and limited binding site. (a) Overall view of the BARF1-hCSF-1 binding interface. BARF1 DE $\beta$-turn and $B C$ loop $C \alpha$ atoms are represented as spheres, and hCSF-1 helices are annotated. (b) Superposition of BARF1-hCSF-1 onto the hCSF-1-hCSF-1R molecular envelope as determined by $\mathrm{EM}^{20}$, through their common hCSF-1. (c) Structural details of the BARF1-hCSF-1 interaction site and selected interactions. A BARF1-rhBARF sequence alignment indicates $D E \beta$-turn and $B C$ loop conservation. Involved hCSF-1 residues are annotated onto the hCSF-1 sequence, and the boxed regions indicate the dimer interface region. Interacting residues are labeled, and hydrogen bonds are drawn as dashed lines. Prime symbol (') denotes residues in the second subunit of the hCSF-1 dimer.

and Supplementary Table 3). To our surprise, mutating residues Arg82, Ser83, Asn85 and Thr86 from the DE $\beta$-turn, which contributes only side chain interactions to the binding site, to alanine had virtually no effect on the thermodynamics of the interaction (Supplementary Fig. 2a and Supplementary Table 3). Thus, the BC loop main chain contacts mediated by Val38, Ser39, Leu40 and Gly41 appear to provide most of the binding energy for the nanomolar affinity between the BC-loop mutant variant and hCSF-1. The BC loop side chain interactions then likely contribute another $3.4 \mathrm{kcal} \mathrm{mol}^{-1}$ (four orders of magnitude gain in affinity) to reach the low picomolar affinity of the BARF1-hCSF-1 complex. This corollary also agrees well with the preservation of the $\mathrm{BC}$ loop and $\mathrm{DE} \beta$-turn conformations in unbound BARF1. The importance of the BC loop and DE $\beta$-turn is further illustrated by the strict conservation of the two corresponding sequences in rhesus LCV BARF1 (BARF1 $1_{\text {rhLCV }}$ ) (Fig. 2c), which shares an overall 75\% sequence identity with BARF1. Indeed, the BARF $1_{\text {rhLCV }}-\mathrm{hCSF}-1$ interaction reveals a nearly identical binding fingerprint as for the canonical BARF1-hCSF-1 complex (Supplementary Fig. 2b and Fig. 3a).

To gain further insights into the apparent lack of steric overlap between the BARF1-binding site on hCSF-1 and the cognate receptorbinding site, we employed a previously reported quadruple point mutant of hCSF-1 carrying mutations in two discrete interaction patches crucial for hCSF-1R binding (H9A H15A and Q58A D59A $)^{23,27}$. Binding studies by ITC show that this quadruple point mutant of hCSF-1 has a nearly identical thermodynamic profile to wild-type hCSF-1 (Supplementary Fig. 2c), further supporting the steric distinctness of the BARF1-hCSF-1 binding site.

\section{BARF1 is an allosteric decoy receptor for hCSF-1}

It has been unclear whether BARF1 is a decoy receptor of hCSF-1 or rather a co-stimulatory molecule ${ }^{13,15}$. A role as decoy receptor is seemingly incompatible with the distinct BARF1 binding site on hCSF-1 (Fig. 2b). On the other hand, the picomolar affinity for the BARF1hCSF-1 interaction reported here is typical for decoy receptors ${ }^{28}$.

To investigate the postulated agonistic or antagonistic role of BARF1, we sought to exploit the very slow dissociation kinetics of the BARF1-hCSF-1 interaction (Fig. 3c) in an ITC experiment, whereby purified BARF1-hCSF-1 was titrated with the full-length ectodomain of hCSF-1R (hCSF-1 $\left.\mathrm{R}_{\mathrm{D} 1-\mathrm{D} 5}\right)$. The titration reveals that when hCSF-1 is bound by BARF1, it is unable to interact with its cognate receptor (Fig. 4a), sharply contrasting with the high-affinity interaction revealed in control titrations of hCSF- $1 \mathrm{R}_{\mathrm{D} 1-\mathrm{D} 5}$ against hCSF-1 $\left(K_{\mathrm{D}}=\right.$ $92 \mathrm{nM}$ ) (Supplementary Fig. 3a) and vice versa ${ }^{20}$. This result further validates our quantification of the BARF1-hCSF-1 interaction by displacement ITC (Fig. 3b).

We further wondered whether the apparent combined neutralization and inactivation of hCSF-1 by BARF1 could be reflected in a comparative cellular assay that would investigate stimulation of purified CD14 ${ }^{+}$ monocytes from human peripheral blood mononuclear cells (PBMCs) as a function of three molecular species: hCSF-1, BARF1 and purified BARF1-hCSF-1 complex. Western blot analysis of hCSF-1R activation, as traced via trans-phosphorylation of Tyr723 (pTyr723) on hCSF-1R and of cellular hCSF-1R, revealed that the BARF1-hCSF-1 complex is unable to signal via hCSF-1R (Fig. 4b). This contrasts sharply with normal hCSF-1 signaling, whereby hCSF-1R shows a dose-dependent response and saturation as a function of hCSF-1 stimulation, followed by internalization and degradation (Fig. 4 b).

A follow-up cellular assay employing the CSF-1-dependent Bac1.2F5 cell line ${ }^{29}$ showed that cell growth proceeds until BARF1 abundance exceeds a 1:3 stoichiometric ratio with hCSF-1 (Fig. 4c and Supplementary Fig. 3b). Similarly, cellular proliferation is initially suppressed in the presence of a fixed amount of BARF1 and at rising concentrations of hCSF-1 until a molar excess of hCSF-1 is reached.

BARF1 blocks cooperative assembly of hCSF-1R-hCSF-1

The lack of structural overlap between the BARF1-hCSF-1 and hCSF-1RhCSF-1 interaction sites and the ability of BARF1 to efficiently 

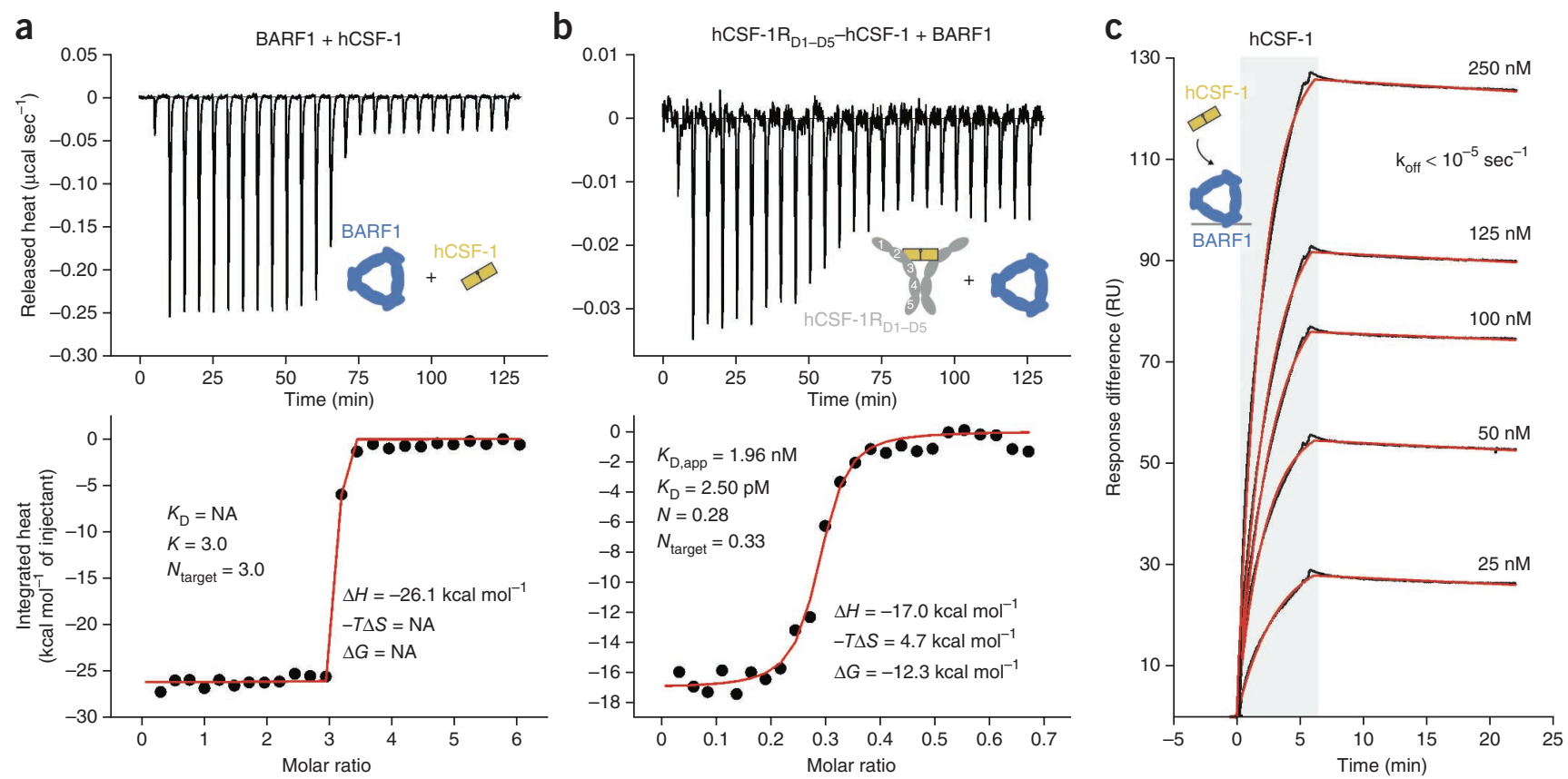

Figure 3 BARF1 sequesters hCSF-1 with ultrahigh affinity. (a,b) Thermodynamic analysis of the BARF1-hCSF-1 interaction. All thermogram titles and schematic molecular representations correspond to the experimental geometry 'cell contents + syringe contents'. For calculation of the stoichiometry, individual proteins were considered in their functional oligomeric state: that is, hexameric BARF1, dimeric hCSF-1 and monomeric hCSF-1R $\mathrm{R}_{\mathrm{D} 1-\mathrm{D} 5}$. Thermodynamic binding parameters are annotated below the traces (NA, not applicable). (c) Kinetic profile of the BARF1-hCSF-1 interaction by SPR measurements. Immobilized BARF1 was flowed with rising concentrations of hCSF-1 (gray shaded region), after which injection was halted. Sensorgrams are fitted according to a 1:1 model, and an approximation for the hCSF-1 dissociation rate constant is indicated.

abrogate hCSF-1 signaling prompted us to explore a mechanistic explanation that goes beyond antagonization based on steric hindrance.

A crystallographic study on a fortuitous new crystal form of hCSF-1 revealed five independent copies of hCSF-1 in the asymmetric unit of the crystal (Table 1). The five hCSF-1 dimers feature an intrinsic butterfly-like plasticity about their dimer interface, manifested by different tilt angles between hCSF-1 subunits about a pivot point at the top of the hCSF-1 dimer interface (Fig. 5a). This location essentially constitutes the focal point of the BARF1-binding site. Superposition of the 13 hCSF- 1 structures at our disposal-that is, the five copies of unbound hCSF-1 and the eight copies of hCSF-1 derived in the two crystal forms of the BARF1-hCSF-1 complex-establishes that BARF1 locks hCSF-1 into a single conformational species corresponding to the most collapsed state in our set of hCSF-1 structures (Fig. 5a). Indeed, when compared to the most open state of unbound hCSF-1, the BARF1-bound state collapses by $10^{\circ}$. Such butterfly-like domain swings of cytokine subunits, both in extent and direction, appear to be a common structural feature among the dimeric $\alpha$-helical cytokines of the RTK-III family of receptors, when involved in a ternary complex with the full-length ectodomains of their cognate receptors $^{30,31}$ (Supplementary Fig. 4). Furthermore, conserved dimerization-sequence fingerprints in extracellular-domain D4 have been linked to the ability of RTK-III to engage in homotypic receptor-receptor interactions ${ }^{20,30}$. In the case of hCSF-1R, such receptor-receptor interactions were shown to contribute one order of magnitude to the total affinity of the extracellular assembly ${ }^{20,32}$, calling for a mechanistic scenario based on positive cooperativity.

To investigate the mechanistic importance of cytokine subunit plasticity in the assembly of a cooperative complex, we tested the ability of hCSF-1 $\mathrm{R}_{\mathrm{D} 1-\mathrm{D} 3}$, which comprises the complete ligand-binding portion of CSF-1R but lacks the D4-D5 module necessary for homotypic receptor contacts, to engage in an encounter complex with hCSF-1 bound to BARF1. An ITC experiment whereby BARF1-hCSF-1 complex was titrated with hCSF-1 $\mathrm{R}_{\mathrm{D} 1-\mathrm{D} 3}$ revealed a low-affinity binding $\left(K_{\mathrm{D}}=3 \mu \mathrm{M}\right)$ that is about ten times weaker than the interaction of hCSF-1 $\mathrm{R}_{\mathrm{D} 1-\mathrm{D} 3}$ with free hCSF-1 (ref. 20) (Fig. 5a). Furthermore, the interaction is based on a binary complex (one hCSF-1 $\mathrm{R}_{\mathrm{D} 1-\mathrm{D} 3}$ and one BARF1-bound hCSF-1) instead of a ternary complex, which would be expected from the capacity of free hCSF-1 to bivalently engage two molecules of hCSF-1 $\mathrm{R}_{\mathrm{D} 1-\mathrm{D} 3}$ (ref. 20). Therefore, this provides the first evidence to date that the inherent plasticity of the hCSF-1 dimer must be critically important for the assembly of the extracellular complex.

It would thus appear that BARF1 forces dimeric hCSF-1 to adopt a conformation that is too constrained to evoke a ternary cytokinereceptor complex yet exposed enough to form a binary encounter complex with an hCSF-1R variant lacking domains 4 and 5. Furthermore, the inability of the full-length ectodomain of hCSF-1R to engage in any kind of interaction with BARF1-bound hCSF-1 (Fig. 4a) suggests that the plasticity of the dimeric cytokine is inextricably linked to extracellular complex formation including an encounter complex and homotypic receptor contacts.

To investigate this principle further, we constructed a monomeric hCSF-1 variant (Supplementary Fig. 5a,b) and used ITC to characterize its ability to bind to hCSF-1 $\mathrm{R}_{\mathrm{D} 1-\mathrm{D} 5}$, hCSF-1 $\mathrm{R}_{\mathrm{D} 1-\mathrm{D} 3}$ (Fig. $5 \mathbf{b}$ ) and BARF1 (Supplementary Fig. 5b). hCSF-1 $\mathrm{R}_{\mathrm{D} 1-\mathrm{D} 5}$ and hCSF-1 $\mathrm{R}_{\mathrm{D} 1-\mathrm{D} 3}$ both bind monomeric hCSF-1 with a stoichiometry of 1.0 to form a binary complex that is similar in affinity $\left(K_{\mathrm{D}}=\sim 2.5 \mu \mathrm{M}\right)$ to the complex of hCSF-1 $\mathrm{R}_{\mathrm{D} 1-\mathrm{D} 3}$ with BARF1-bound hCSF-1 (Fig. 5a). We note that these affinities are lower by a factor of $10^{2}$ and $10^{1}$, respectively, than the canonical $K_{\mathrm{D}}$ values of these ectodomain variants against dimeric hCSF-1 (Supplementary Fig. 5c and ref. 20). This essentially means that the contribution of dimeric hCSF-1 to the 

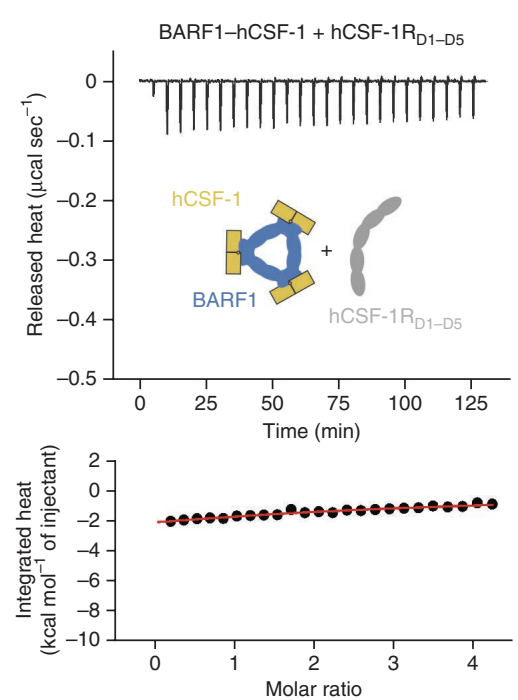

b

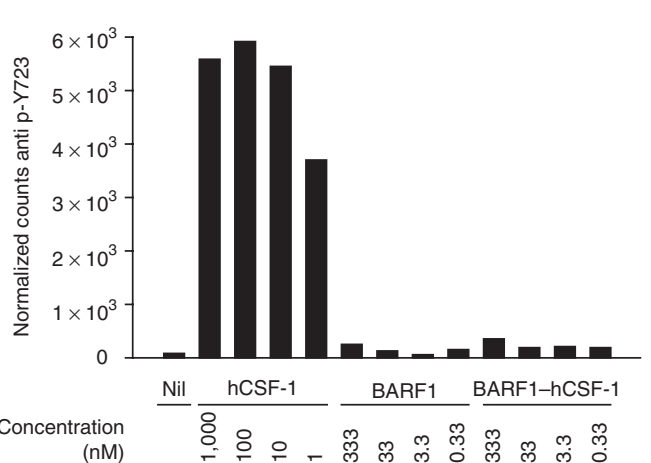

C BARF1 titration against fixed hCSF-1 concentration

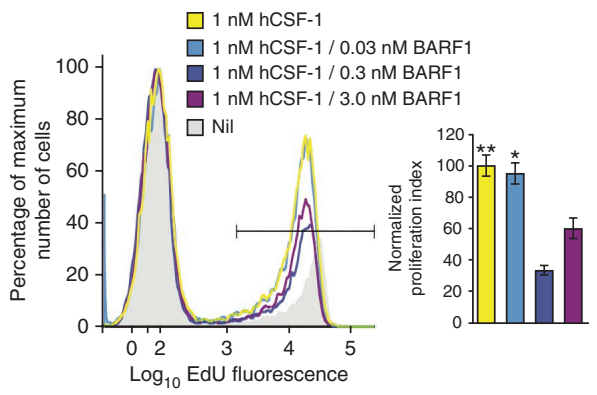

hCSF-1 titration against fixed BARF1 concentration

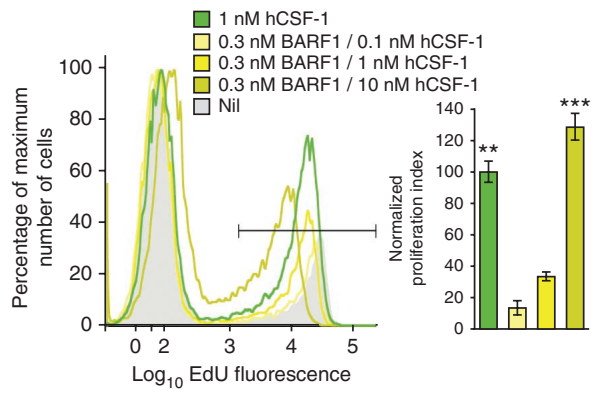

Figure 4 BARF1 is a decoy receptor for hCSF-1 and blocks maturation of monocytes into macrophages. (a) Preformed and isolated BARF1-hCSF-1 complex titrated with hCSF-1R (b) Stimulation of human PBMC monocytes with hCSF-1 and corresponding stoichiometric amounts of BARF1 or BARF1-hCSF-1. The boxed panels correspond to a western blot analysis of hCSF-1R activation via anti-pTyr723 antibodies and hCSF-1R cellular presence via anti-CSF-1R antibodies. (c) CSF-1-dependent Bac1.2F5 cells were stimulated with hCSF-1, BARF1 or varying stoichiometric ratios of both (color coded; shaded gray area, basal Bac1.2F5 proliferation). The horizontal bars identify cells that proliferated (measured by incorporation of 5-ethynyl-2'-deoxyuridine (EdU)). The insets show the normalized proliferation indices from seven independent experiments ( \pm s.e.m.) where ${ }^{*},{ }^{* *}$ and ${ }^{* * *}$, respectively, indicate a $P$ value of $\leq 0.05,0.01$ and 0.001 relative to the untreated control, by Kruskal-Wallis test.

cooperative hCSF-1-hCSF-1R complex is nearly as much as that from receptor-receptor interactions. This also confirms the prevailing notion that dimeric hCSF-1 is needed for ternary complex formation and therefore receptor activation. At the same time, monomeric hCSF-1 is unable to bind to BARF1 (Supplementary Fig. 5b), confirming that the hCSF-1 dimer interface is the actual target for BARF1.

\section{Mouse CSF-1 is an unexpected ligand for BARF1}

EBV infection is limited to humans and New World monkeys, yet many studies have reported a mitogenic role of BARF1 in a murine cellular background $^{8,9,11,33}$. Given the strong conservation of the BARF1 interaction site on hCSF-1 in mouse CSF-1 (mCSF-1) (Supplementary Fig. 6a), we sought to assess the possible interaction of BARF1 with mCSF-1. Unexpectedly, the interaction of BARF1 with recombinant mCSF-1 is also characterized by a subnanomolar $K_{\mathrm{D}}$ and slow dissociation kinetics (Fig. 6a and Supplementary Fig. 6b), which at first sight contrasts with the reported inability of BARF1 to neutralize mCSF-1 (ref. 15).

To investigate the structural basis of the BARF1-mCSF-1 interaction, we determined the crystal structure of the BARF1-mCSF-1 complex to 4.5 - $\AA$ resolution (Table 1). The BARF1-mCSF- 1 structure is overall very comparable to the BARF1-hCSF-1 complex (r.m.s. deviation of $0.6 \AA$ for BARF1 $\mathrm{C} \alpha$ atoms; r.m.s. deviation of $0.8 \AA$ a

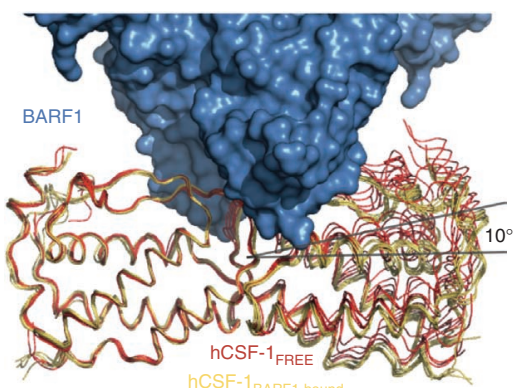

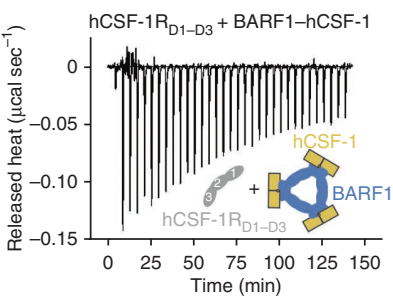

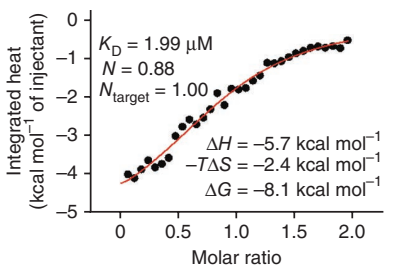

b
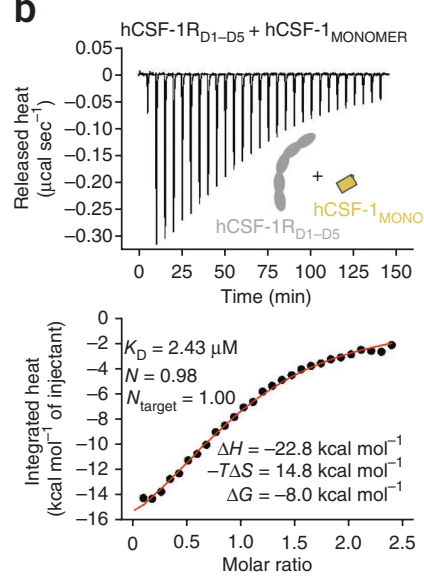
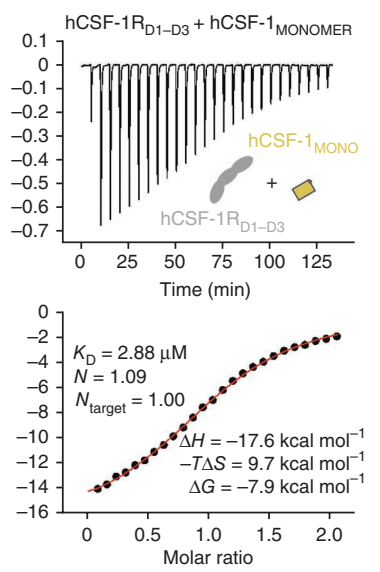

Figure 5 BARF1 abrogates formation of a cooperative hCSF-1R-hCSF-1 ternary complex. (a) Unbound (red) and BARF1-bound (yellow) hCSF-1 molecules are superposed with one hCSF-1 monomer as alignment reference. BARF1 (blue) induces a compacting tilt of $10^{\circ}$ onto the hCSF-1 protomers upon binding. Right, titration of hCSF-1R $\mathrm{D}_{\mathrm{D} 1-\mathrm{D} 3}$ with preformed BARF1-hCSF-1 complex. (b) Titration of hCSF-1R $\mathrm{D}_{1-D 5}$ and hCSF-1R $\mathrm{R}_{\mathrm{D} 1-\mathrm{D} 3}$ with mutant monomeric hCSF-1. 
a
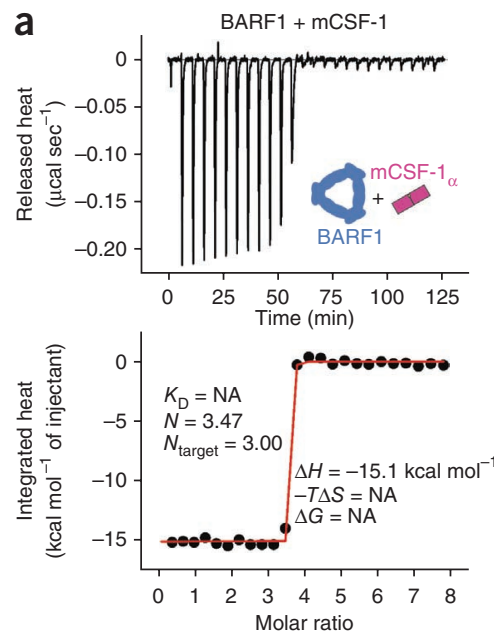
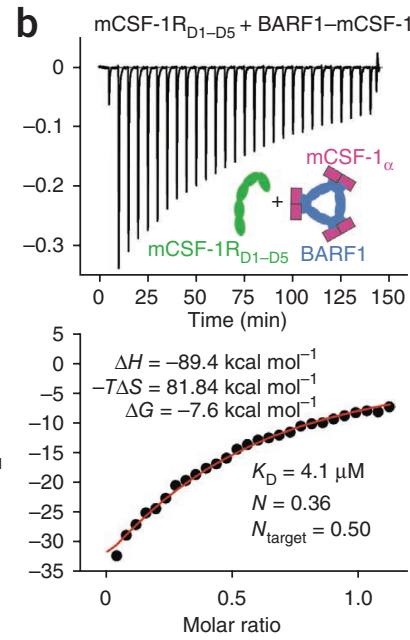
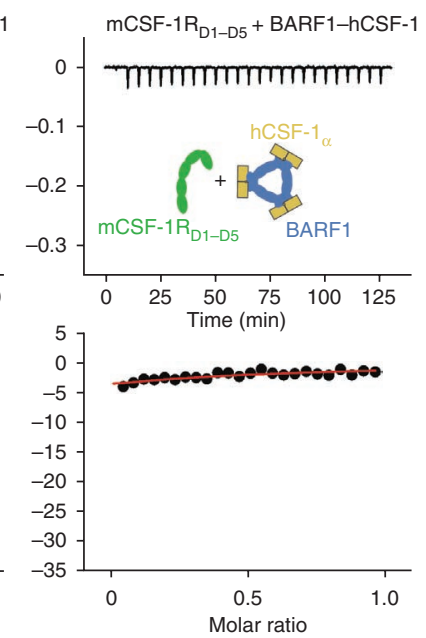

C

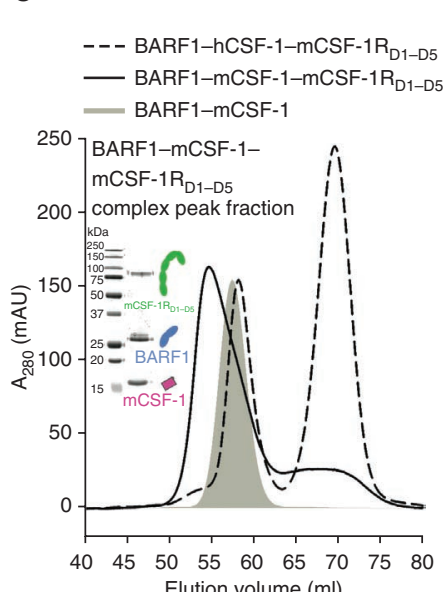

Figure 6 BARF1 can target mCSF-1 but does not abrogate a mCSF-1 $\mathrm{R}_{\mathrm{D} 1-\mathrm{D} 5}-\mathrm{mCSF}-1$ signaling complex. (a,b) Thermodynamic analysis of the BARF1-mCSF-1 interaction. All thermogram titles and schematic molecular representations correspond to the experimental geometry 'cell contents + syringe contents'. Thermodynamic binding parameters are annotated below the traces (NA, not applicable). (c) Isolation of the BARF1-mCSF-1-mCSF-1 $R_{\text {D1-D5 }}$ higher-order complex by SEC after ITC. The peak shape is consistent with a micromolar affinity complex, and there is slight dissociation of the complex into BARF1-mCSF-1 and mCSF-1R D1-D5 $_{\text {components. BARF1-hCSF-1 }}$ and mCSF-1 $R_{D 1-D 5}$ elute separately after ITC. The inset shows a stained SDS-PAGE strip corresponding to the

BARF1-mCSF-1-mCSF-1 $\mathrm{R}_{\mathrm{D} 1-\mathrm{D} 5}$ peak fraction. (d) Equilibrium SPR measurements of the interactions of BARF1-mCSF-1 (left) and BARF1-hCSF-1 (right) with mCSF-1R

for $\mathrm{C} \alpha$ atoms in bound hCSF-1 and mCSF-1) in that it shows the same binding stoichiometry, overall organization of the toroidal structure and involvement of the BC loop and DE $\beta$-turn (Supplementary Fig. 6c). We also determined the crystal structure of unbound mCSF-1 (Table 1 and Supplementary Fig. 6d), to investigate the conformational plasticity of mCSF-1 upon binding
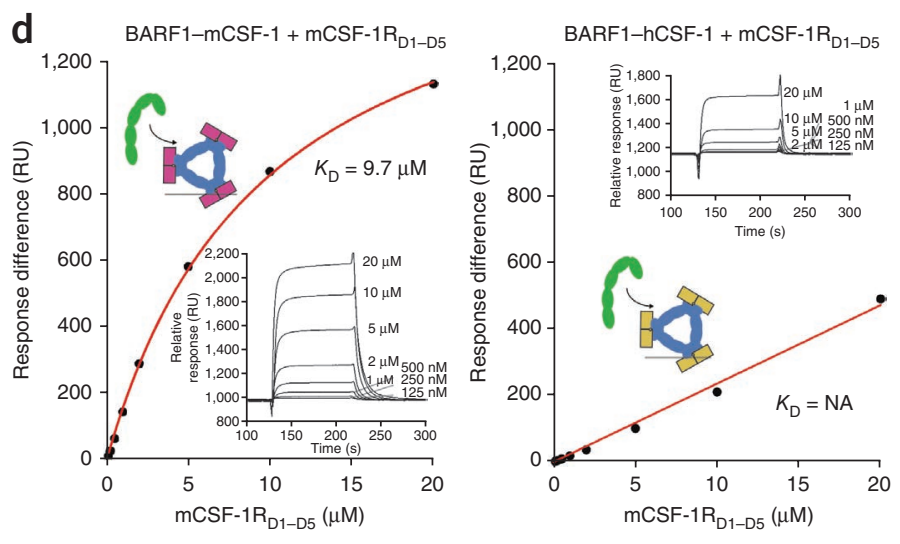

to BARF1. The intersubunit tilt in unbound mCSF-1 is identical to that observed in the binary complex with mCSF- $1 \mathrm{R}_{\mathrm{D} 1-\mathrm{D} 3}$ (ref. 23) (r.m.s. deviation of $0.6 \AA$ for C $\alpha$ atoms) (Supplementary Fig. 6d), consistent with the observation that the cytokine intersubunit tilt is only featured in cytokine-RTKIII ternary complexes (Supplementary Fig. 4). In addition, unbound $\mathrm{mCSF}-1$ is $5^{\circ}$ more open than unbound hCSF-1 (Supplementary Fig. 6d). Therefore, whereas BARF1 enforces the same type of tilt to mCSF-1 and hCSF-1 alike, mCSF-1
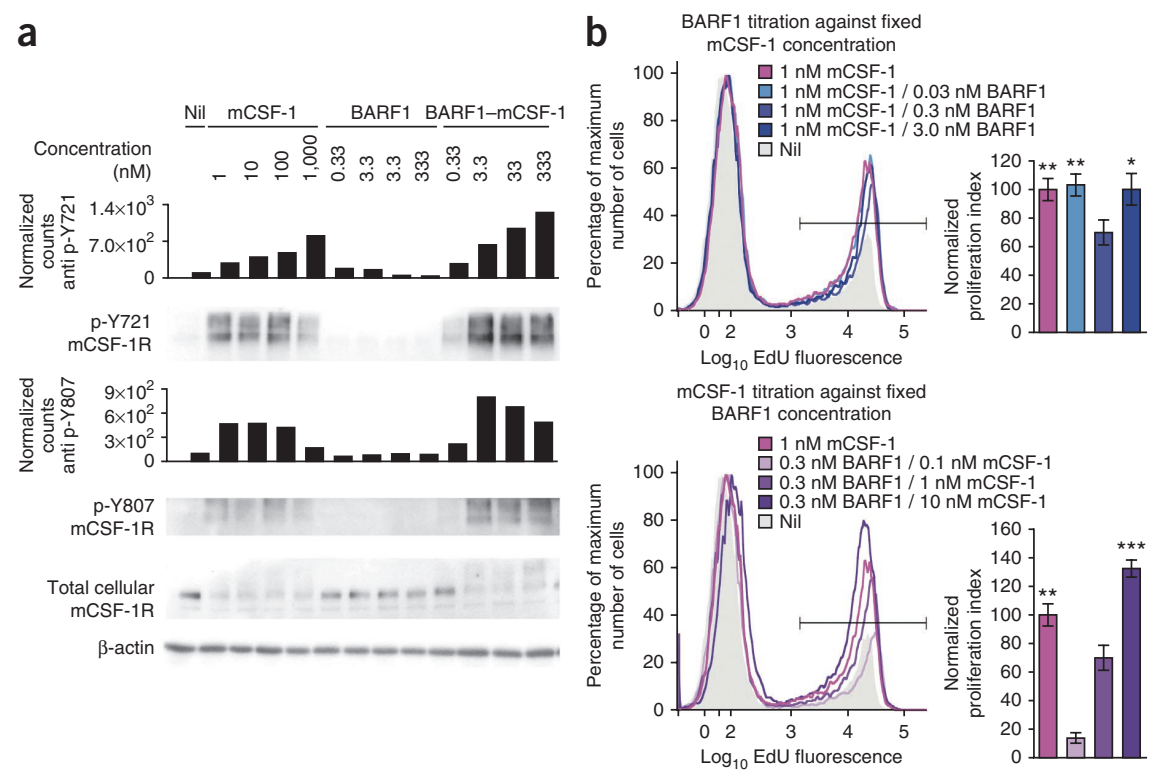

Figure 7 BARF1 does not block activation of mCSF-1R by mCSF-1. (a) Stimulation of mouse bone-marrow monocytes with mCSF-1 and corresponding stoichiometric amounts of BARF1 or BARF1-mCSF-1. The graphs show densitometric evaluation of mCSF-1R kinase-domain activation and cellular presence via anti-phosphoY721 (anti p-Y721), antiphosphoY807 (anti p-Y807)and anti-CSF-1R antibodies. (b) CSF-1-dependent Bac1.2F5 cells were stimulated with mCSF-1, BARF 1 or varying stoichiometric ratios of both (color coded; shaded gray area, basal Bac1.2F5 proliferation). The horizontal bars identify cells that proliferated $\left(\mathrm{EdU}^{+}\right)$. The insets show the normalized proliferation indices from seven independent experiments ( \pm s.e.m.) where *, ${ }^{* *}$ and ${ }^{* * *}$, respectively, indicate $P$ values of $\leq 0.05,0.01$ and 0.001 relative to the untreated control, by Kruskal-Wallis test. 
undergoes a comparatively larger structural transition $\left(15^{\circ}\right.$ versus $10^{\circ}$ tilt) (Supplementary Fig. 6c and Fig. 5a).

BARF1 does not block signaling mediated by mCSF-1R-mCSF-1 To test whether BARF1-bound mCSF- 1 could still bind to its cognate receptor, $\mathrm{mCSF}-1 \mathrm{R}$, preformed $\mathrm{BARF} 1-\mathrm{mCSF}-1$ complex isolated by size-exclusion chromatograpy (SEC) was titrated into mCSF-1 $\mathrm{R}_{\mathrm{D} 1-\mathrm{D} 5}$. In marked contrast to the corresponding setup involving the human counterparts (Fig. 4a), mCSF-1 bound to BARF1 does bind to mCSF$1 \mathrm{R}_{\mathrm{D} 1-\mathrm{D} 5}$ (Fig. 6b). The BARF1-mCSF-1-mCSF-1 $\mathrm{R}_{\mathrm{D} 1-\mathrm{D} 5}$ interaction is characterized by an unusually high enthalpic component $(\sim-90 \mathrm{kcal}$ $\mathrm{mol}^{-1}$ ), which is $\sim 40 \mathrm{kcal} \mathrm{mol}^{-1}$ more favorable than the enthalpic contribution measured for the cognate mCSF-1-mCSF-1 $\mathrm{R}_{\mathrm{D} 1-\mathrm{D} 5}$ interaction $^{20}$. Yet, notably, the interaction is $\sim 200$ times weaker, $4.1 \mu \mathrm{M}$ versus $22 \mathrm{nM}^{20}$, as a result of a large entropic penalty $(-80$ $\mathrm{kcal} \mathrm{mol}^{-1}$ ). Furthermore, the measured stoichiometry is indicative of a mCSF-1-mCSF-1 $\mathrm{R}_{\mathrm{D} 1-\mathrm{D} 5}$ ternary complex.

Taking advantage of the cross-reactivity of hCSF- 1 and $\mathrm{mCSF}-1 \mathrm{R}^{20}$, we titrated BARF1-hCSF-1 into mCSF-1 $\mathrm{R}_{\mathrm{D} 1-\mathrm{D} 5}$ in an ITC experiment and found that BARF1 abolishes binding of hCSF-1 to mCSF$1 \mathrm{R}_{\mathrm{D} 1-\mathrm{D} 5}$ (Fig. 6b). Thus, BARF1 has evolved to mechanistically target hCSF-1 specifically and not mCSF-1. In addition, whereas the assembly principles of human and mouse CSF-1-CSF-1R complexes are similar $^{20}$, the modularity requirements and plasticity of human and mouse CSF-1 must differ in the context of their cooperative complexes with cognate receptors. Given the low micromolar affinity for the BARF1-mCSF-1-mCSF-1R $\mathrm{D}_{\text {1-D5 }}$ supercomplex, we wondered whether such assembly could be isolated by SEC. Indeed, loading the post-ITC titration mixture of BARF1-mCSF-1-mCSF-1 $\mathrm{R}_{\mathrm{D} 1-\mathrm{D} 5}$ led to the isolation of the supercomplex, whereas the post-ITC titration mixture of mCSF-1 $\mathrm{R}_{\mathrm{D} 1-\mathrm{D} 5}$ and BARF1-hCSF-1 yielded molecular species that eluted separately (Fig. 6c).

In a parallel surface plasmon resonance (SPR) experiment, immobilized BARF1-mCSF-1 and BARF1-hCSF-1 complexes were allowed to interact with mCSF-1 $\mathrm{R}_{\mathrm{D} 1-\mathrm{D} 5}$. $\mathrm{mCSF}-1 \mathrm{R}_{\mathrm{D} 1-\mathrm{D} 5}$ showed significant binding to the BARF1-mCSF-1 complex $\left(K_{\mathrm{D}}=9.7 \mu \mathrm{M}\right)$ (Fig. 6d), in good agreement with the $K_{\mathrm{D}}=4.1 \mu \mathrm{M}$ value obtained via ITC (Fig. 6b). Whereas some signal could be observed for the interaction of mCSF$1 \mathrm{R}_{\mathrm{D} 1-\mathrm{D} 5}$ with the BARF1-hCSF-1 complex, the SPR sensorgrams suggest a $K_{\mathrm{D}}$ for the interaction well into the millimolar range. Notably, the interaction of $\mathrm{mCSF}-1 \mathrm{R}_{\mathrm{D} 1-\mathrm{D} 3}$ and $\mathrm{BARF} 1-\mathrm{mCSF}-1$ reveals a $K_{\mathrm{D}}=12.5 \mu \mathrm{M}$ and is essentially indistinguishable from the interaction with the full-length ectodomain (Supplementary Fig. 7).

To extrapolate our findings to a cellular context, we set up an assay similar to the BARF1-hCSF-1 interaction, whereby isolated mouse bone-marrow cells containing progenitors expressing $\mathrm{mCSF}-1 \mathrm{R}$ were stimulated with either mCSF-1, BARF1 or the BARF1-mCSF-1 complex. Stimulation with BARF1-mCSF-1 complex led to strong activation of mCSF-1R, as evidenced by the native-like phosphorylation status of Tyr721 and Tyr807 of mCSF-1R (Fig. 7a), in stark contrast to our cellular assays investigating activation of hCSF-1R in the context of the BARF1-hCSF-1 complex (Fig. 4b). In addition, mCSF-1-driven proliferation in Bac1.2F5 cells could not be antagonized in the presence of BARF1 (Fig. $7 \mathbf{b}$ and Supplementary Fig. 3b).

Together, our data show that the low micromolar affinity measured for BARF1-bound mCSF-1 for its cognate mCSF-1R ectodomain in vitro is likely improved by three orders of magnitude in a cellular context involving full-length receptor, as was already observed for several RTK-III complexes ${ }^{20,31,32,34}$. Thus, the interaction of mCSF-1R with mCSF- 1 in the context of a BARF1-mCSF-1 complex is strong enough to stimulate cellular proliferation, and, depending on the internalization kinetics of the BARF1-mCSF-1mCSF-1R supercomplexes, it may lead to overstimulation and tumorigenic transformation, in line with previous work in mouse model systems.

\section{DISCUSSION}

Two decades after the discovery that EBV secretes the immunomodulatory protein BARF1 and the subsequent emergence of BARF1 as an omnipresent viral protein in EBV-associated malignancies, we present the molecular and structural basis of sequestration and inactivation of the essential human hematopoietic cytokine hCSF-1 by the secreted decoy receptor BARF1. Prior to our study, diverse studies on the structural basis of immune subversion by immune modulators encoded by large DNA viruses ${ }^{35-39}$ had established that molecular mimicry ${ }^{3}$, whereby the viral molecule resembles its human counterpart structurally and functionally, lies at the heart of such virushuman protein complexes.

Perhaps the most far-reaching dimension of our work is the uncovering of a unique mechanism for the inactivation of a human growth factor by a viral decoy receptor to achieve immunomodulation. BARF1 is structurally different from the cognate receptor for hCSF-1, and it evolved to target a novel binding site at the dimer interface of hCSF-1, distinct from the cognate receptor-binding site. Via this binding strategy, BARF1 forces hCSF-1 to adopt a conformational state that renders the cytokine unable to establish a ternary complex with its cognate receptor, thereby abrogating signaling. Thus, not only is BARF1 an exquisitely efficient decoy receptor, albeit a nonclassical one, it is essentially an allosteric competitive antagonist as well. This unprecedented (to our knowledge) mechanistic conclusion provides a substantive advancement in our understanding of the possible molecular mechanisms deployed by pathogens in evading and subverting key host signaling pathways. Importantly, it frees us from the expectation that molecular mimicry and direct competition against common interaction sites should be at play. This is particularly important at a time when studies delineating the interactome landscape of major human pathogens take center stage ${ }^{40,41}$.

A second mechanistic breakthrough from our studies is the discovery that the plasticity of dimeric hCSF-1 is a mechanistic prerequisite for establishing signaling-competent ternary complexes with hCSF1R. Up until now, cooperative assembly of the hCSF-1-hCSF-1R ternary complex was thought to proceed as a result of receptor-receptor interactions following formation of an initial cytokine-receptor encounter complex. Not only have we now quantified the affinity of this basic encounter complex, but we have also determined that hCSF-1 dimer plasticity contributes one order of magnitude to the affinity of the extracellular complex. Thus, a synthesis of experimental evidence now allows the deconvolution of the in vivo affinity of the CSF-1CSF-1R complex ${ }^{25}(\sim 50 \mathrm{pM})$ into modular contributions stemming from an initial encounter complex, the dimer plasticity of hCSF-1, ectodomain receptor contacts ${ }^{20}$, TM-domain dimerization ${ }^{42}$ and the two-dimensional spatial confinement of the membrane. In a viral context, the ubiquity of circulating hCSF-1 (ref. 43) and its picomolar affinity to its cognate receptor essentially necessitate the employment of a competitive decoy receptor that can establish a tight and longlived complex with hCSF-1. Furthermore, our studies indicate that an encounter complex, cytokine intersubunit plasticity and receptorreceptor contacts all are concomitant events in the cooperative mechanism of hCSF-1R activation. Such insights bring us closer to understanding the intricacies of cytokine-induced receptor activation and are likely to initiate a renewed interest in mechanistic studies of other oligomeric cytokines and receptor families. 
Our studies aiming to investigate the fortuitous BARF1-mCSF-1 interaction clearly caution against the study of LCV or EBV pathogenesis and isolated BARF1 in non-native cellular backgrounds. Although mouse and human CSF-1 fulfill the same role in vivo in their cognate context, EBV exclusively infects humans and New World monkeys. Yet BARF1, like many other protein effectors, has been extensively studied in immortalized mouse cell lines such as NIH/3T3 or Balb/c3T3 (refs. 8,12,33). These studies attributed a mitogenic and transforming activity to BARF1, results that cannot be reconciled with observations in isolated PBMC monocytes and B cells ${ }^{13,15}$ and rhesus macaques infected with rhesus $\mathrm{LCV}^{14}$. The unexpected finding that BARF1-bound mCSF-1 can potently activate mCSF-1R-keeping in mind the corollary that monocytes may be driven to transformation due to overstimulation-is likely a consequence of a number of factors: (i) the distinctness of the BARF1 binding site on CSF-1 away from the cognate receptor-binding sites, (ii) the species differences in human and mouse CSF-1R activation ${ }^{20}$ and (iii) the evolutionary optimization of BARF1 to inactivate hCSF-1.

Finally, the identification of a new functional and modulatory hot spot centered at the intersubunit disulfide of CSF-1 has created profound implications for the 'druggability' and therapeutic targeting of the dimer interface region of CSF-1 and the homologous helical hematopoietic cytokines IL-34, stem-cell factor and Flt3 ligand. Efforts to antagonize RTK-III have predominantly focused on kinase-domain inhibition, an approach prone to poor specificity and development of resistance to treatment. In addition, the fortuitous picomolar affinity of mouse CSF-1 for BARF1 and its poor cross-reactivity with human CSF- $1 \mathrm{R}^{20}$ may render mouse CSF-1 an attractive starting point for the development of a BARF1 antagonist in vitro or in vivo. Likewise, BARF1 and BARF $1_{\mathrm{rhLCV}}$ could serve as lead protein-based therapeutics for the clinical targeting of the neutralization of CSF-1 mediated signaling.

\section{METHODS}

Methods and any associated references are available in the online version of the paper.

Accession codes. Atomic coordinates and structure factors are deposited in the Protein Data Bank, with accession codes 3UEZ (BARF1hCSF-1 complex to $3.4-\AA$ resolution), 4ADF (BARF1-hCSF-1 complex to $4.4-\AA$ resolution), 4ADQ (BARF1-mCSF-1 complex to 4.5-Å resolution), 3UF2 (hCSF-1 to 2.75- $\AA$ resolution), 3UF5 (mCSF-1 to 2.8 - $\AA$ resolution).

Note: Supplementary information is available in the online version of the paper.

\section{ACKNOWLEDGMENTS}

We thank the Swiss Light Source (SLS), the European Synchrotron Radiation Facility (ESRF) and the Deutsches Elektronen-Synchrotron/European Molecular Biology Laboratory (DESY/EMBL) for synchrotron-beam time allocation and the staff of beamlines X06SA and X06DA (SLS), ID-29 and ID14-3 (ESRF) and X33 (DESY/EMBL) for technical support. Access to these synchrotron facilities is supported by the European Commission under the Seventh Framework Programme: Research Infrastructures, grant agreement number 226716. This research project was supported by grants from the Research Foundation Flanders (FWO) (3G064307, G059710 and G0B7912N) and Ghent University (BOF instrument) to S.N.S.; a Bundesministerium für Bildung und Forschung (BMBF) research grant Sync-Life (Contract: 05K10YEA) to D.I.S.; an ANR-MIME-2006 grant to N.T. and W.P.B.; and an FWO-Odysseus grant to B.N.L. J.E. would like to acknowledge the joint 2011 APS/CCP4 School for assistance with crystallographic structure refinement. We thank the SWITCH Laboratory at VIB-Belgium for access to FFF-MALS facilities and assistance during data collection and analysis. We also thank F. Wang (Harvard University, Boston, Massachusetts, USA) for providing cDNA of rhBARF1 and Christian Gorba (EMBL Hamburg, Hamburg, Germany) for help with the NMA refinement. I.G. thanks the EM platform of the
Partnership for Structural Biology (Grenoble) for access to EM equipment. J.E., K.V. and B.V. are supported as research fellows of the FWO. P.P. is a Marie Curie (FP7) post-doctoral fellow.

\section{AUTHOR CONTRIBUTIONS}

J.E. and N.B. expressed and purified recombinant proteins, with contributions from A.B. J.E. carried out all crystallographic studies with contributions from N.B., A.B. and S.N.S. J.E., N.B. and B.V. carried out ITC with contributions from S.N.S. N.T. and W.P.B. carried out preliminary structural studies on independently produced material. J.E., N.B. and B.V. carried out SPR studies with contributions from N.T. and W.P.B. K.V. provided tools and assistance for tissue culture. P.P. and B.N.L. carried out cellular assays. J.E., A.V.S. and D.I.S. carried out SAXS studies. I.G. carried out EM imaging and data analysis. J.E., N.B., B.V. and S.N.S. established the experimental approach and designed experiments. S.N.S. directed the study. J.E. and S.N.S. wrote the manuscript with contributions from all authors.

\section{COMPETING FINANCIAL INTERESTS}

The authors declare no competing financial interests.

Published online at http://www.nature.com/doifinder/10.1038/nsmb.2367.

Reprints and permissions information is available online at http://www.nature.com/ reprints/index.html.

1. Young, L.S. \& Rickinson, A.B. Epstein-Barr virus: 40 years on. Nat. Rev. Cancer 4, 757-768 (2004)

2. Kutok, J.L. \& Wang, F. Spectrum of Epstein-Barr virus-associated diseases Annu. Rev. Pathol. 1, 375-404 (2006).

3. Alcami, A. Viral mimicry of cytokines, chemokines and their receptors. Nat. Rev. Immunol. 3, 36-50 (2003).

4. Rivailler, P., Cho, Y.G. \& Wang, F. Complete genomic sequence of an Epstein-Barr virus-related herpesvirus naturally infecting a new world primate: a defining point in the evolution of oncogenic lymphocryptoviruses. J. Virol. 76, 12055-12068 (2002).

5. Seto, E., Ooka, T., Middeldorp, J. \& Takada, K. Reconstitution of nasopharyngeal carcinoma-type EBV infection induces tumorigenicity. Cancer Res. 68, 1030-1036 (2008).

6. Seto, E. et al. Epstein-Barr virus (EBV)-encoded BARF1 gene is expressed in nasopharyngeal carcinoma and EBV-associated gastric carcinoma tissues in the absence of lytic gene expression. J. Med. Virol. 76, 82-88 (2005).

7. Orlova, N., Fogg, M.H., Carville, A. \& Wang, F. Antibodies to lytic infection proteins in lymphocryptovirus-infected rhesus macaques: a model for humoral immune responses to Epstein-Barr virus infection. Clin. Vaccine Immunol. 18, 1427-1434 (2011).

8. Wei, M.X. \& Ooka, T. A transforming function of the BARF1 gene encoded by Epstein-Barr virus. EMBO J. 8, 2897-2903 (1989)

9. Wei, M.X., Moulin, J.C., Decaussin, G., Berger, F. \& Ooka, T. Expression and tumorigenicity of the Epstein-Barr virus BARF1 gene in human Louckes B-lymphocyte cell line. Cancer Res. 54, 1843-1848 (1994).

10. Wei, M.X., deTurenne-Tessier, M., Decaussin, G., Benet, G. \& Ooka, T. Establishment of a monkey kidney epithelial cell line with the BARF1 open reading frame from Epstein-Barr virus. Oncogene 14, 3073-3081 (1997).

11. Sheng, W., Decaussin, G., Ligout, A., Takada, K. \& Ooka, T. Malignant transformation of Epstein-Barr virus-negative Akata cells by introduction of the BARF1 gene carried by Epstein-Barr virus. J. Virol. 77, 3859-3865 (2003).

12. Sall, A. et al. Mitogenic activity of Epstein-Barr virus-encoded BARF1 protein. Oncogene 23, 4938-4944 (2004).

13. Cohen, J.I. \& Lekstrom, K. Epstein-Barr virus BARF1 protein is dispensable for B-cell transformation and inhibits alpha interferon secretion from mononuclear cells. J. Virol. 73, 7627-7632 (1999).

14. Ohashi, M., Orlova, N., Quink, C. \& Wang, F. Cloning of the Epstein-Barr virusrelated rhesus lymphocryptovirus as a bacterial artificial chromosome: a loss-offunction mutation of the rhBARF1 immune evasion gene. J. Virol. 85, 1330-1339 (2011).

15. Strockbine, L.D. et al. The Epstein-Barr virus BARF1 gene encodes a novel, soluble colony-stimulating factor-1 receptor. J. Virol. 72, 4015-4021 (1998).

16. Warren, M.K. \& Ralph, P. Macrophage growth factor CSF-1 stimulates human monocyte production of interferon, tumor necrosis factor, and colony stimulating activity. J. Immunol. 137, 2281-2285 (1986).

17. Lin, J.C., Zhang, Z.X., Chou, T.C., Sim, I. \& Pagano, J.S. Synergistic inhibition of Epstein-Barr virus: transformation of $B$ lymphocytes by $\alpha$ and $\gamma$ interferon and by 3'-azido-3'-deoxythymidine. J. Infect. Dis. 159, 248-254 (1989).

18. Hislop, A.D., Taylor, G.S., Sauce, D. \& Rickinson, A.B. Cellular responses to viral infection in humans: lessons from Epstein-Barr virus. Annu. Rev. Immunol. 25 587-617 (2007).

19. Hamilton, J.A. Colony-stimulating factors in inflammation and autoimmunity. Nat. Rev. Immunol. 8, 533-544 (2008).

20. Elegheert, J. et al. Extracellular complexes of the hematopoietic human and mouse CSF-1 receptor are driven by common assembly principles. Structure 19, 1762-1772 (2011) 
21. Tarbouriech, N., Ruggiero, F., de Turenne-Tessier, M., Ooka, T. \& Burmeister, W.P. Structure of the Epstein-Barr virus oncogene BARF1. J. Mol. Biol. 359, 667-678 (2006).

22. Gorba, C., Miyashita, O. \& Tama, F. Normal-mode flexible fitting of high-resolution structure of biological molecules toward one-dimensional low-resolution data. Biophys. J. 94, 1589-1599 (2008).

23. Chen, X., Liu, H., Focia, P.J., Shim, A.H. \& He, X. Structure of macrophage colony stimulating factor bound to FMS: diverse signaling assemblies of class III receptor tyrosine kinases. Proc. Natl. Acad. Sci. USA 105, 18267-18272 (2008).

24. Velazquez-Campoy, A. \& Freire, E. Isothermal titration calorimetry to determine association constants for high-affinity ligands. Nat. Protoc. 1, 186-191 (2006).

25. Roussel, M.F., Downing, J.R., Rettenmier, C.W. \& Sherr, C.J. A point mutation in the extracellular domain of the human CSF-1 receptor (c-fms proto-oncogene product) activates its transforming potential. Cell 55, 979-988 (1988).

26. Lupardus, P.J., Birnbaum, M.E. \& Garcia, K.C. Molecular basis for shared cytokine recognition revealed in the structure of an unusually high affinity complex between IL-13 and IL-13R $\alpha 2$. Structure 18, 332-342 (2010).

27. Taylor, E.W., Fear, A.L., Bohm, A., Kim, S.H. \& Koths, K. Structure-function studies on recombinant human macrophage colony-stimulating factor (M-CSF). J. Biol. Chem. 269, 31171-31177 (1994).

28. Mantovani, A., Locati, M., Vecchi, A., Sozzani, S. \& Allavena, P. Decoy receptors: a strategy to regulate inflammatory cytokines and chemokines. Trends Immunol. 22, 328-336 (2001).

29. Morgan, C., Pollard, J.W. \& Stanley, E.R. Isolation and characterization of a cloned growth factor dependent macrophage cell line, BAC1.2F5. J. Cell. Physiol. 130, 420-427 (1987).

30. Yuzawa, S. et al. Structural basis for activation of the receptor tyrosine kinase KIT by stem cell factor. Cell 130, 323-334 (2007).

31. Verstraete, K. et al. Structural insights into the extracellular assembly of the hematopoietic Flt3 signaling complex. Blood 118, 60-68 (2011).
32. Ma, X. et al. Structural basis for the dual recognition of helical cytokines IL-34 and CSF-1 by CSF-1R. Structure 20, 676-687 (2012).

33. Sheng, W., Decaussin, G., Sumner, S. \& Ooka, T. N-terminal domain of BARF1 gene encoded by Epstein-Barr virus is essential for malignant transformation of rodent fibroblasts and activation of BCL-2. Oncogene 20, 1176-1185 (2001).

34. Lemmon, M.A., Pinchasi, D., Zhou, M., Lax, I. \& Schlessinger, J. Kit receptor dimerization is driven by bivalent binding of stem cell factor. J. Biol. Chem. 272 6311-6317 (1997).

35. Krumm, B., Meng, X., Li, Y., Xiang, Y. \& Deng, J. Structural basis for antagonism of human interleukin 18 by poxvirus interleukin 18-binding protein. Proc. Natl. Acad. Sci. USA 105, 20711-20715 (2008).

36. Liu, H. et al. Structural basis of semaphorin-plexin recognition and viral mimicry from Sema7A and A39R complexes with PlexinC1. Cell 142, 749-761 (2010).

37. Nuara, A.A. et al. Structure and mechanism of IFN-gamma antagonism by an orthopoxvirus IFN-gamma-binding protein. Proc. Natl. Acad. Sci. USA 105, 1861-1866 (2008).

38. Yang, Z., West, A.P. Jr. \& Bjorkman, P.J. Crystal structure of TNF $\alpha$ complexed with a poxvirus MHC-related TNF binding protein. Nat. Struct. Mol. Biol. 16, 1189-1191 (2009).

39. Yoon, S.I., Jones, B.C., Logsdon, N.J. \& Walter, M.R. Same structure, different function: crystal structure of the Epstein-Barr virus IL-10 bound to the soluble IL-10R1 chain. Structure 13, 551-564 (2005).

40. Calderwood, M.A. et al. Epstein-Barr virus and virus human protein interaction maps. Proc. Natl. Acad. Sci. USA 104, 7606-7611 (2007).

41. Uetz, P. et al. Herpesviral protein networks and their interaction with the human proteome. Science 311, 239-242 (2006).

42. Finger, C., Escher, C. \& Schneider, D. The single transmembrane domains of human receptor tyrosine kinases encode self-interactions. Sci. Signal. 2, ra56 (2009).

43. Janowska-Wieczorek, A. et al. Increased circulating colony-stimulating factor- 1 in patients with preleukemia, leukemia, and lymphoid malignancies. Blood 77, 1796-1803 (1991) 


\section{ONLINE METHODS}

Production of recombinant CSF-1- and CSF-1R-ectodomain variants. Recombinant h-mCSF-1 (residues 1-149) were expressed in Escherichia coli and purified from inclusion bodies after in vitro refolding ${ }^{20}$. h-mCSF- $1 \mathrm{R}_{\mathrm{D} 1-\mathrm{D} 5}$ and h-mCSF- $1 R_{D 1-D 3}$ CSF- $1 \mathrm{R}$ variants were produced in transiently transfected HEK293T cells in the presence of kifunensine ${ }^{20,44}$. Human and mouse CSF-1 $\mathrm{R}_{\mathrm{D} 1-\mathrm{D} 5^{-}}$ CSF-1 and CSF-1 $\mathrm{R}_{\mathrm{D} 1-\mathrm{D} 3}-\mathrm{CSF}-1$ complexes were isolated by size-exclusion chromatography (SEC) after incubation of CSF-1R ectodomains with a molar excess of purified cognate CSF-1.

Production of recombinant BARF1 and BARF1-CSF-1 complexes. BARF1 (residues 1-221) was expressed via the pHLsec vector ${ }^{45}$ in transiently transfected HEK293T cells in the presence of kifunensine ${ }^{44}$. BARF1 was purified by metal affinity chromatography (Talon FF, Clontech) followed by SEC. BARF1-h-mCSF-1 complexes were isolated by SEC after incubation of BARF1 with a molar excess of purified h-mCSF-1. The molecular masses of purified BARF1, h-mCSF-1 and the BARF1-h-mCSF-1 complexes were estimated by MALS (DAWN HELEOS, Wyatt) following fractionation by field-flow fractionation (FFF).

Crystallization and X-ray data collection. All crystallization experiments were conducted using the sitting-drop vapor-diffusion geometry using a Mosquito robot (TTP LabTech). Recombinant hCSF-1 was crystallized in $0.1 \mathrm{M} \mathrm{MgCl}_{2}, 0.1 \mathrm{M}$ sodium citrate $\mathrm{pH} 5.0$ and $15 \% \mathrm{w} / \mathrm{v}$ PEG 4000. Crystals of recombinant mCSF-1 were grown from $0.2 \mathrm{M} \mathrm{CaCl}_{2}, 0.1 \mathrm{M}$ Tris, $\mathrm{pH}$ 8.0, 20\% PEG 6000. Crystals were incubated in stabilization buffer supplemented with $20 \%$ glycerol for $\mathrm{X}$-ray data collection. BARF1-hCSF-1 was crystallized in two related conditions: (i) $0.1 \mathrm{M}$ Tris- $\mathrm{HCl}, \mathrm{pH} 8.50,1.0 \mathrm{M}$ ammonium sulfate and (ii) $0.1 \mathrm{M}$ Tris $\mathrm{HCl}$, $\mathrm{pH}$ 8.50, 1.0 M ammonium phosphate. Crystals of BARF1-mCSF-1 were obtained from 0.1 M HEPES, pH 7.5, 0.6 M sodium citrate. Crystals were incubated in stabilization buffer supplemented with $25 \%$ ethylene glycol for X-ray data collection. Diffraction data were collected at beamlines X06DA of the SLS (hCSF-1, BARF1-hCSF-1) and ID-29 of the ESRF (mCSF-1, BARF1-mCSF-1) and were processed with the XIA2 routine ${ }^{46}$.

Crystallographic structure determination and refinement. The crystal structure of hCSF-1 in space group $P 2_{1}$ was determined via maximum-likelihood molecular replacement (MR) implemented in Phaser ${ }^{47}$ using the deposited $\mathrm{C} \alpha$ coordinates of hCSF-1 (PDB code $1 \mathrm{HMC})^{48}$. Crystals of hCSF-1 satisfied the unit-cell relation $c \cos \beta=-a / 2$ and were pseudomerohedrally twinned emulating a $C 222_{1}$ metric symmetry. Model building was carried out using Arp/Warp ${ }^{49}$ and manually completed using $\operatorname{Coot}^{50}$. Crystallographic refinement was carried out with Phenix ${ }^{51}$. The twinning operator $(h,-k,-h-l)$ was applied in the final rounds of refinement. The structure of unbound $\mathrm{mCSF}-1$ was determined by MR using coordinates derived from the mCSF- $1 \mathrm{R}_{\mathrm{D} 1-\mathrm{D} 3}-\mathrm{mCSF}-1$ complex (PDB code $3 \mathrm{EJJ})^{23}$ and was refined with Phenix.

The crystal structures of BARF1-hCSF-1 (crystal form 1) and BARF1-mCSF-1 were determined by MR using the structure of unbound BARF1 (PDB code $2 \mathrm{CH} 8)^{21}$ and the herein-determined crystal structures of hCSF- 1 and mCSF- 1 . The respective crystal asymmetric units contain two h-mCSF-1 dimers and four BARF1 monomers. The complete BARF1-h-mCSF-1 complex can be generated by a three-fold symmetry operation about the unit-cell $c$ axis. For crystal form 2 of the BARF1-hCSF-1 complex, MR indicated the presence of two complete complexes in the asymmetric unit. The structure was determined using the refined structure of BARF1-hCSF-1 to 3.4- $\AA$ resolution. Refinement of the BARF1-hCSF-1 structure (crystal form 1) to 3.4- $\AA$ resolution was carried out using Phenix with local NCS restraints. Initial refinement of BARF1-hCSF-1 to 4.4- $\AA$ resolution (crystal form 2) was performed with Refmac5 using weighted reference-structure restraint $s^{52}$ derived from the high-resolution hCSF-1 and BARF1 models, and local NCS restraints. Model building was facilitated by $\mathrm{B}$ factor-sharpened maps ${ }^{52}$, which revealed many side chains and complete $\mathrm{Man}_{5-9} \mathrm{GlCNAC}_{2}$ glycan trees. Subsequent refinement of BARF1-hCSF-1 to $4.4-\AA$ resolution and refinement of BARF1-mCSF-1 to $4.5-\AA$ resolution was carried out with Phenix.

Small-angle X-ray scattering. Data were collected at beamline X33 of the EMBL (DESY, Hamburg) at $298 \mathrm{~K}$ within a momentum transfer range of $0.01 \AA^{-1}<$ $s<0.6 \AA^{-1}$ where $s=4 \pi \sin (\theta) / \lambda$ and $2 \theta$ is the scattering angle. The BARF1 and BARF1-hCSF-1 samples were measured at several solute concentrations (1-10 mg ml${ }^{-1}$ in $20 \mathrm{mM}$ HEPES, pH 7.50, $150 \mathrm{mM} \mathrm{NaCl}$ and $50 \mathrm{mM} \mathrm{NaPO}_{4}$, $\mathrm{pH} 7.40,100 \mathrm{mM} \mathrm{NaCl}$, respectively) with intermittent buffer solution. Data reduction, invariant calculation and calculation of X-ray scattering patterns from structural models were carried out using protocols implemented in the ATSAS suite $^{53}$. NMA of BARF1 was performed using the NOMAD-REF server ${ }^{54}$; SAXS data were fit using an iterative fitting protocol ${ }^{22}$ using a linear combination of two out of the ten lowest-frequency normal modes.

Electron microscopy. Purified BARF1-h-mCSF-1 complexes $\left(\sim 0.05 \mathrm{mg} \mathrm{ml}^{-1}\right)$ were applied to a carbon-mica interface and stained with $2 \%(\mathrm{w} / \mathrm{v})$ sodium silicotungstate at $\mathrm{pH}$ 7.0. Images were recorded under low-dose conditions with a JEOL-1200-EX-II microscope at $100 \mathrm{kV}$ and nominal 40,000× magnification. Selected negatives were digitized on a Zeiss scanner (Photoscan TD) (step size $14 \mu \mathrm{m}$, pixel size $3.5 \AA$ A). BARF1-h-mCSF-1 complex particles were selected interactively $\left(45 \times 10^{3}\right.$ subframes of $80 \times 80$ pixels $)$ using EMAN ${ }^{55}$, CTFcorrected $^{56}$, and low-path-filtered at $15 \AA$. Subsequent data processing was performed with Imagic-5 (ref. 57). The translationally centered data set was subjected to multivariate statistical analysis and classification to obtain a set of references for multireference alignment. Class averages after cycles of multireference alignment, multivariate statistical analysis and classification ( 220 particles per class) were compared to projections of the crystal structures of BARF1-hCSF-1 and BARF1-mCSF-1 filtered to 25 - $\AA$ resolution.

Human peripheral blood mononuclear cell (PBMC) stimulation. PBMC monocytes were isolated from collected blood on a Ficoll gradient ${ }^{58}$ and $\mathrm{CD} 14^{+}$ PBMC monocytes were enriched (Miltenyi Biotec). Cells were maintained in RPMI 10\% human AB serum containing Gentamicin $\left(0.05 \mathrm{mg} \mathrm{ml}^{-1}\right)$ for $18 \mathrm{~h}$ to increase hCSF-1R expression and reduce receptor internalization due to endogenous hCSF-1 $\left(1 \times 10^{6}\right.$ cells ml $\left.{ }^{-1}\right)$ and were subsequently stimulated with hCSF-1, BARF1 or their complex for $2 \mathrm{~min}$ at $37^{\circ} \mathrm{C}$. The reaction was stopped by addition of ice-cold PBS. Cells were pelleted and lysed in lysis buffer (40 mM Tris- $\mathrm{HCl}, 275 \mathrm{mM} \mathrm{NaCl}, 20 \%$ glycerol, $1 \mathrm{mM} \mathrm{PMSF}, 20 \mathrm{mg} \mathrm{ml}^{-1}$ aprotinin, $20 \mathrm{mg} \mathrm{ml}^{-1}$ leupeptin, $2 \mathrm{mM}$ sodium orthovanadate, $50 \mathrm{mM}$ sodium fluoride) for $15 \mathrm{~min}$ at $4^{\circ} \mathrm{C}$ under agitation. Cell lysate was clarified by centrifugation. Samples were subjected to SDS-PAGE and immunoblotted using anti-pCSF-1R (pTyr723) (Cell Signaling Technology, cat. no. 3155S, clone 49C10; 1:1,000), anti-CSF-1R (Cell Signaling Technology, cat. no. 3152S; 1:1,000) or anti-GAPDH (Sigma-Aldrich, cat. no. G9545; 1:10,000) antibodies.

Mouse bone-marrow mononuclear cell stimulation. Bone-marrow cells were extracted from the femurs and tibias of 6-8-week-old C57BL/6 mice ${ }^{59}$. After red-blood-cell osmotic lysis, bone-marrow cells were counted and plated overnight at $3 \times 10^{6}$ cells per condition in $3 \mathrm{ml}$ of culture medium (RPMI 1640,10\% FCS, $0.05 \mathrm{mg} \mathrm{ml}^{-1}$ gentamicin and $5 \mu \mathrm{M} \beta$-mercaptoethanol). Cells were subsequently stimulated with mCSF-1, BARF1 or their complex in $1 \mathrm{ml}$ for $2 \mathrm{~min}$ at $37^{\circ} \mathrm{C}$. The reaction was stopped by addition of ice-cold PBS.

Bac1.2F5 proliferation. Bac1.2F5 cells ${ }^{29}$ were cultured and seeded at $0.25 \times 10^{6}$ cells per well in a six-well plate in starving medium (without CSF-1) for $24 \mathrm{~h}$ before the described stimulations. After stimulation for $24 \mathrm{~h}$ with h-mCSF-1, BARF1 or their stoichiometric mixtures, cellular proliferation was evaluated by the EdU method ${ }^{60}$ ( $50 \mathrm{mM}$ EdU for $2 \mathrm{~h}$ followed by staining via the Clickit reaction (Invitrogen)). Viability was assessed by the green LIVE/DEAD dye (Invitrogen). Cells were analyzed by flow cytometry (LSR II, Becton Dickinson), and a minimum of 20,000 live cells were recorded and analyzed. Graphical depictions were produced for one experiment representative of seven independent experiments. Analyses were performed on the gate indicated in the graphs. The normalized proliferation index is defined as control-blanked percentage of proliferating cells normalized to the proliferation from $1 \mathrm{nM} \mathrm{h}$-mCSF-1. Statistical analysis was performed using a Kruskal-Wallis test (GraphPad Prism 5), and the data were annotated accordingly when displaying $P$ values of $\leq 0.05$, 0.01 or 0.001 .

Surface plasmon resonance. For BARF1 flowed with h-mCSF-1, BARF1 (50 $\mu \mathrm{g}$ $\mathrm{ml}^{-1}$ in $10 \mathrm{mM}$ sodium acetate, $\mathrm{pH} 4.0$ ) was coupled to an activated CM5 chip to $\sim 500$ responsive units (RU), with $\sim 500 \mathrm{RU}$ of aldolase as negative control. h-mCSF-1 
$(25,50,100,125,250 \mathrm{nM})$ diluted in HBS-EP buffer was injected $\left(15 \mu 1 \mathrm{~min}^{-1}\right)$ for $5 \mathrm{~min}$, followed by dissociation for $25 \mathrm{~min}$. Surface regeneration was performed using pulses of $0.1 \mathrm{M} \mathrm{NaOH}$ followed by a recovery phase. The sensorgrams were fitted to a simple 'one-to-one' model accounting for mass-transfer effects. For BARF1-h-mCSF-1 flowed with h-mCSF-1R $\mathrm{D}_{\mathrm{D} 1-\mathrm{D} 3 / 5}, \mathrm{BARF1}$ was coupled to an activated CM5 chip to $\sim 5000 \mathrm{RU}$ and was saturated with h-mCSF-1 (to $\sim 1000 \mathrm{RU}$ ). The negative-control cell was coupled with $\sim 5000 \mathrm{RU}$ of BARF1. h-mCSF-1R $\mathrm{R}_{\mathrm{D} 1-\mathrm{D} 5}$ or h-mCSF-1 $\mathrm{R}_{\mathrm{D} 1-\mathrm{D} 3}(125 \mathrm{nM}, 250 \mathrm{nM}, 500 \mathrm{nM}, 1 \mu \mathrm{M}, 2 \mathrm{uM}, 5 \mathrm{uM}, 10 \mu \mathrm{M}, 20 \mu \mathrm{M})$ diluted in HBS-EP buffer was injected $\left(10 \mu \mathrm{min}^{-1}\right)$ onto the BARF1-h-mCSF-1coupled chip for $90 \mathrm{~s}$, followed by dissociation for $200 \mathrm{~s}$.

Isothermal titration calorimetry. Prior to all measurements (VP-ITC MicroCalorimeter, 310K), the buffer of all protein samples was matched to HBS buffer by SEC. Protein concentrations were measured spectrophotometrically at $280 \mathrm{~nm}$ using theoretical extinction coefficients. An initial injection of $3 \mu \mathrm{l}$ was followed by injections of $10 \mu \mathrm{l}$ applied $300 \mathrm{~s}$ apart. The thermal titration data were analyzed and fit (Origin-ITC) to a 'one-binding-site model,' and the apparent molar enthalpy $\left(\Delta H^{\circ}\right)$, apparent entropy $\left(\Delta S^{\circ}\right)$, association constant $\left(K_{\mathrm{A}}\right)$ and stoichiometry of binding $(N)$ were determined. Several titrations were performed to evaluate reproducibility.

44. Chang, V.T. et al. Glycoprotein structural genomics: solving the glycosylation problem. Structure 15, 267-273 (2007).

45. Aricescu, A.R. et al. Eukaryotic expression: developments for structural proteomics. Acta Crystallogr. D. Biol. Crystallogr. 62, 1114-1124 (2006).

46. Winter, G. xia2: an expert system for macromolecular crystallography data reduction. J. Appl. Crystallogr. 43, 186-190 (2010).
47. McCoy, A.J. et al. Phaser crystallographic software. J. Appl. Crystallogr. 40, 658-674 (2007).

48. Pandit, J. et al. Three-dimensional structure of dimeric human recombinant macrophage colony-stimulating factor. Science 258, 1358-1362 (1992).

49. Langer, G., Cohen, S.X., Lamzin, V.S. \& Perrakis, A. Automated macromolecular model building for X-ray crystallography using ARP/wARP version 7. Nat. Protoc. 3, 1171-1179 (2008).

50. Emsley, P., Lohkamp, B., Scott, W.G. \& Cowtan, K. Features and development of Coot. Acta Crystallogr. D. Biol. Crystallogr. 66, 486-501 (2010).

51. Adams, P.D. et al. PHENIX: a comprehensive Python-based system for macromolecular structure solution. Acta Crystallogr. D. Biol. Crystallogr. 66, 213-221 (2010).

52. Nicholls, R.A., Long, F. \& Murshudov, G.N. Low-resolution refinement tools in REFMAC5. Acta Crystallogr. D. Biol. Crystallogr. 68, 404-417 (2012).

53. Petoukhov, M.V. et al. New developments in the ATSAS program package for smallangle scattering data analysis. J. Appl. Crystallogr. 45, 342-350 (2012).

54. Lindahl, E., Azuara, C., Koehl, P. \& Delarue, M. NOMAD-Ref: visualization, deformation and refinement of macromolecular structures based on all-atom normal mode analysis. Nucleic Acids Res. 34, W52-W56 (2006).

55. Ludtke, S.J., Baldwin, P.R. \& Chiu, W. EMAN: Semiautomated software for highresolution single-particle reconstructions. J. Struct. Biol. 128, 82-97 (1999).

56. Mindell, J.A. \& Grigorieff, N. Accurate determination of local defocus and specimen tilt in electron microscopy. J. Struct. Biol. 142, 334-347 (2003).

57. van Heel, M. Harauz, G., Orlova, E.V., Schmidt, R. \& Schatz, M. A new generation of the IMAGIC image processing system. J. Struct. Biol. 116, 17-24 (1996).

58. Fuss, I.J., Kanof, M.E., Smith, P.D. \& Zola, H. Isolation of whole mononuclear cells from peripheral blood and cord blood. Curr. Protoc. Immunol. 85, 7.1.1-7.1.8 (2009).

59. Pouliot, P., Willart, M.A., Hammad, H. \& Lambrecht, B.N. Studying the function of dendritic cells in mouse models of asthma. Methods Mol. Biol. 595, 331-349 (2010).

60. Salic, A. \& Mitchison, T.J. A chemical method for fast and sensitive detection of DNA synthesis in vivo. Proc. Natl. Acad. Sci. USA 105, 2415-2420 (2008). 